\title{
Efeito do Início de Ocorrência do Míldio na Produção de Frutos do Meloeiro
}

\author{
A. Apoliano dos Santos, J. Emilson Cardoso, Julio C. Vidal, F. Marto P. Viana \& Adroaldo G. Rossetti \\ Embrapa Agroindústria Tropical, Cx. Postal 3761, CEP 60511-110, Fortaleza, CE, e-mail: apoliano@cnpat.embrapa.br
}

(Aceito para publicação em 17/06/2003)

Autor para correspondência: A. Apoliano dos Santos

SANTOS, A.A., CARDOSO, J.E., VIDAL, J.C., VIANA, F.M.P. \& ROSSETTI, A.G. Efeito do início de ocorrência do míldio na produção de frutos do meloeiro. Fitopatologia Brasileira 28:548-551. 2003.

\section{RESUMO}

O míldio, causado por Pseudoperonospora cubensis, é a principal doença fúngica da cultura do meloeiro (Cucumis melo) no período chuvoso nas regiões produtoras do Nordeste brasileiro, e para o seu controle são realizadas até dez aplicações de fungicidas. $\mathrm{O}$ estabelecimento de sistemas de previsão de danos causados por esta doença requer uma estimativa sob várias situações epidêmicas. Os danos causados pelo míldio à produção de frutos do meloeiro, levandose em consideração o início do aparecimento dos sintomas e o estádio de desenvolvimento da planta, foram determinados em três experimentos de campo. Utilizou-se a aplicação de uma das misturas fungicidas tiofanato metílico + clorotalonil ou metalaxyl + mancozeb no estabelecimento de menores intensidades de míldio. Houve uma significativa redução do rendimento de frutos quando a doença teve início aos 24 e 36 dias, porém, quando a doença iniciou-se aos 47 dias, nenhum efeito na produção foi observado. Concluiu-se que os aspectos relacionados com a duração e, notadamente, com o início da epidemia, em relação ao ciclo do hospedeiro, devem ser considerados no estabelecimento de modelos de prognósticos de danos à produção.

Palavras-chave adicionais: Pseudoperonospora cubensis, Cucumis melo, perdas.

\section{ABSTRACT}

\section{Effect of the downy mildew initiation on the melon fruit production}

Downy mildew caused by Pseudoperonospora cubensis is the main disease affecting melon (Cucumis melo) fruit during the rainy period in areas of Northeastern Brazil where the crop is produced. Up to ten fungicide sprays are used throughout the crop season. The establishment of risk analysis for forecast of damages caused by this disease requires an estimate of the losses under different epidemic situations. Three field experiments were carried out to determine the damage caused by downy mildew to the fruit yield of the melon crop.
The epidemic was considered to begin in the phenological cycle of the host plant. Different intensities of epidemics were achieved by spraying one of the following fungicide mixtures: methyl thiophanate + chlorothalonil or metalaxyl + mancozeb. There was a significant reduction on the fruit yield when the disease started at 24 and 36 days after planting, but when the disease started on day 47 , no effect in production was observed. It was concluded that the duration of the the epidemic and when it began in relation to the cycle of the host, should be considered when establishing prognostic models to describe the risks of yield losses.
A cultura do meloeiro (Cucumis melo L.) é de grande importância para o Nordeste brasileiro. Condições climáticas, como temperaturas elevadas e altos níveis de insolação, favorecem o desenvolvimento de frutos com elevado teor de sólidos solúveis. O Nordeste destaca-se entre as demais regiões do país, respondendo por $79 \%$ da área plantada com melão no Brasil e mais de $89 \%$ da produção nacional (Anuário, 1999). O cultivo contínuo do meloeiro tem possibilitado a ocorrência, em níveis epidêmicos, de doenças capazes de causar severos danos às plantas, aumentando, conseqüentemente, os custos econômicos e ecológicos da cultura (Rego, 1995; Santosetal., 2000).

O míldio, causado pelo fungo Pseudoperonospora cubensis ( Berk. \& M. A. Curtis) Rostovzev, é uma das mais importantes doenças do meloeiro no Brasil. Além de sua ocorrência endêmica durante o período seco, esta doença limita a produção no período chuvoso. As infecções durante o período endêmico resultam em fonte de inóculo primário para o período epidêmico, quando as condições ambientais são mais favoráveis, principalmente a umidade elevada. O míldio é uma doença de elevada taxa de progresso, cujo inóculo, sob condições climáticas favoráveis (e.g. água - livre nas folhas e umidade relativa elevada), pode dispersar rapidamente no campo (Blancard et al., 1996; Kurozawa \& Pavan, 1997).

A região produtora de melão no Nordeste brasileiro é caracterizada por um período seco alternado por um período chuvoso bem definidos, sendo o primeiro compreendido entre junho a novembro, e o segundo, de dezembro a maio. Os meses mais chuvosos compreendem os de março e abril. O cultivo de meloeiro, apesar de ser realizado durante todo o ano, é feito em sua quase totalidade no período seco (95\%), exatamente em razão do elevado risco de perdas devido ao míldio (Cardoso et al., 2001). Na região do vale do rio Curu, CE, a doença está presente durante todo o ano, embora em intensidades menores no período seco; entretanto, no período chuvoso as condições são altamente favoráveis, inviabilizando a 
Efeito do início de ocorrência do míldio na produção...

exploração econômica do meloeiro. Presentemente, o cultivo de meloeiro nessa região é mínimo. A região da Chapada do Apodi, pelo contrário, produz mais de $30 \%$ da produção de melão do Nordeste no mesmo período, pois o míldio, nessa região, ocorre em menor intensidade, em função do clima menos úmido durante a maior parte do ano, o que permite o manejo químico da doença em condições economicamente viáveis.

Uma estimativa precisa do risco de danos à produção do melão devido ao míldio pode reduzir os custos de controle. A correta estimativa dos danos devido às doenças é necessária para avaliar as estratégias de controle, tomar as decisões mais adequadas sobre os métodos a serem adotados, fazer previsões do rendimento e avaliar as necessidades de pesquisa sobre uma determinada doença (Madden et al., 1981). As variáveis relativas à doença mais usadas para estimativa de danos são a severidade da doença, a área sob a curva de progresso da doença (ASCPD) e a taxa de incremento da doença linearizada ou taxa aparente de infecção (TAI) (James, 1974; Madden, 1983). O uso da porcentagem de danos, baseado no rendimento das parcelas experimentais com menor severidade de doença, tem sido relatada na literatura (Latin, 1992; Latin et al., 1994). Trabalhos anteriores registram danos na produção associadas com doenças do meloeiro (Middleton \& Whitaker, 1946; Thomas, 1998), entretanto, poucos se referem à quantificação desses danos (Latinet al., 1994; Keinath, 2001). Latin (1992), trabalhando com a mancha de alternária [Alternaria cucumerina (Ellis \& Everh.) Elliott] em meloeiro, enfatizou uma limitação ao uso da ASCPD que é a impossibilidade de distinguir entre epidemias de diferentes severidades com valores da ASCPD semelhantes, tais como epidemias leves durante todo o ciclo em relação a epidemias severas que iniciam ao final do ciclo.

Este trabalho teve como objetivo determinar o efeito do início da ocorrência do míldio, considerando o estádio de desenvolvimento da planta, no rendimento do meloeiro.

Os trabalhos de pesquisa foram conduzidos no Campo Experimental do Curu-Paraipaba, da Embrapa Agroindústria Tropical, em Paraipaba, CE e na Fazenda Agrosagno, em Quixeré, CE.

Em Paraipaba, os experimentos foram implantados em duas datas de cultivo: 6 de novembro de 2000 (Experimento A) e 20 de fevereiro de 2001 (Experimento B) enquanto que, em Quixeré, o experimento foi iniciado em 3 de fevereiro de 2001 (Experimento C). Os períodos de novembro a janeiro e de fevereiro a maio representam o início e o pico da estação chuvosa, respectivamente.

A área experimental de Paraipaba é constituída de um Latossolo Amarelo, com textura arenosa, plana e pH 6,2 e, a de Quixeré, de um Cambissolo, com textura média, plana e pH 6,8. Em todos os experimentos, o sistema de irrigação utilizado foi o de gotejadores do tipo "in line".

As fileiras foram, previamente, adubadas com $120 \mathrm{~g}$ de $\mathrm{P}_{2} \mathrm{O}_{5}$, na forma de superfosfato simples $+10 \mathrm{~g}$ de FTE BR 12 $(\mathrm{Zn}=9 \%, \mathrm{~B}=1,8 \%, \mathrm{Cu}=0,8 \%, \mathrm{Fe}=3 \%, \mathrm{Mn}=2 \%$ e $\mathrm{Mo}=0,1 \%)$ $+3 \mathrm{~L}$ de esterco bovino curtido, por metro linear. Duas sementes do híbrido Gold Mine, (Petoseed ${ }^{R}$ Co.) suscetível ao míldio, foram plantadas, manualmente, ao lado de cada gotejador. $\mathrm{O}$ desbaste foi realizado entre sete-dez dias após o plantio deixando-se uma planta por gotejador.

A adubação de cobertura obedeceu a recomendação para a cultura na região (UFC, 1993), sendo feita por meio da irrigação. Esta obedeceu a uma planilha elaborada pela Embrapa Agroindústria Tropical, baseada na evaporação no tanque Classe "A".

O controle preventivo de pragas resumiu-se à aplicação de $2 \mathrm{ml}$ de pyrasophos por litro de água para controle da mosca minadora (Liriomyza sp.), de $3 \mathrm{~g}$ de imidacloprid por litro de água para controle da mosca branca (Bemisia argentifolii Bellows \& Perring, 1994) no primeiro e segundo experimento e de $1,5 \mathrm{ml}$ de endosulfan por litro de água para controle da lagarta das folhas (Diaphania sp.) no terceiro ensaio. Os experimentos foram mantidos livre de plantas daninhas com capinas manuais.

Os tratamentos consistiram de parcelas com controle químico, empregando-se tiofanato metílico $(0,4 \mathrm{~g} / \mathrm{l})+$ chlorothalonil $(1 \mathrm{~g} / \mathrm{l})$, no experimento A, e metalaxyl $(0,24 \mathrm{~g} / \mathrm{l})+$ mancozeb $(1,92 \mathrm{~g} / \mathrm{l})$ nos demais experimentos, e sem controle do míldio. As parcelas experimentais consistiram de aproximadamente 40 plantas, distribuídas em duas fileiras de $10 \mathrm{~m}$, com 20 plantas cada. O espaçamento entre as fileiras foi de $2 \mathrm{~m}$. As parcelas foram casualizadas dentro de cada um dos quatro blocos.

Os fungicidas foram aplicados, semanalmente, a partir do inicio da epidemia, via pulverizador costal manual de 201 , bico J-12 cônico, com componentes D3-25, proporcionando um volume de 600 a 900 1/ha. Uma fileira disseminadora, não tratada, foi deixada entre cada parcela.

A severidade do míldio foi anotada, semanalmente, a partir da primeira detecção, por meio de uma escala diagramática visual de severidade variando de 0 a 4 , consistindo de $0=$ sem sintoma, $1=0,1$ a 10\% da área foliar afetada; $2=11$ a 25\% da área foliar afetada; 3 = 26 a 50\% da área foliar afetada e 4 = acima de $50 \%$ da área foliar afetada. Os valores de severidade por parcela foram transformados em $\sqrt{x+0,5}$ para as análises posteriores.

As curvas de progresso da doença e os seus respectivos modelos ajustados foram determinados. A TAI linearizada (Campbell \& Madden, 1990) e a ASCPD foram estimadas por epidemia (parcela) e comparadas por experimento. As curvas de progresso da doença foram elaboradas para cada um dos três experimentos, considerando-se duas epidemias para cada experimento, uma com controle e outra sem controle químico.

Os frutos de valor comercial ( $\geq 9^{\circ}$ Brix) foram colhidos no estágio ideal de maturação (Dusi, 1992), contados e pesados por parcela. O tratamento com controle químico foi considerado como padrão zero para cálculo de danos.

Os primeiros sintomas de míldio foram observados aos 36, 24 e 47 dias após o plantio nos experimentos A, B e C, respectivamente. Conseqüentemente, o número de aplicações nesses experimentos foi de cinco, seis e três, respectivamente. $\mathrm{O}$ ciclo das plantas nos experimentos variou de 65 a 70 dias, considerando a última colheita. 
No experimento A, observou-se um atraso no início da doença em relação ao experimento $\mathrm{B}$, isso ocorreu porque as condições climáticas no primeiro experimento (A) foram menos favoráveis do que no experimento B. A aplicação de fungicida retardou o início da epidemia em oito dias em relação às parcelas não pulverizadas; entretanto, no segundo experimento o controle foi total, uma vez que se utilizou uma mistura de fungicidas mais eficiente sob as condições climáticas do experimento B, destacando-se a precipitação pluvial $(450 \mathrm{~mm}$ no experimento B contra $80 \mathrm{~mm}$ no experimento A) que propiciou o maior acúmulo de água livre nas folhas. Nas parcelas com aplicação de fungicidas, tanto a ASCPD, como a TAI foram menores do que as parcelas sem aplicação de fungicidas. No experimento C (Quixeré - 03/02/2001), a epidemia iniciou-se aos 47 dias nas parcelas sem aplicação de fungicidas e aos 54 dias, nas parcelas tratadas. Conseqüentemente, tanto a ASCPD (Figura 1A) como a TAI (Figura 1B) foram menores do que nas outras epidemias (aos 24 e 36 dias). Latin (1992), trabalhando com a mancha de alternária em meloeiro, causada por Alternaria cucumerina (Ellis \& Everth) J. A. Elliot. enfatizou uma limitação ao uso da ASCPD, que é a impossibilidade de distinguir entre epidemias de diferentes severidades com valores da ASCPD semelhantes, tais como epidemias leves durante todo o ciclo em relação a epidemias severas que iniciam ao final do ciclo. Esta limitação não foi constatada neste trabalho, uma vez que pelos dados apresentados, os parâmetros usados, revelaram-se bons descritores das epidemias.

No estudo das regressões da ASCPD e da TAI sobre o início da epidemia (em dias após o plantio) obteve-se um maior ajuste $\left(\mathrm{R}^{2}=0,986\right)$ da ASCPD em relação à TAI $\left(\mathrm{R}^{2}=\right.$ 0,863) (Figura 1). Estes dados confirmam a associação negativa entre o tempo do início da manifestação da doença e as variáveis ASCPD e TAI relativas.

A análise de variância dos dados de incidência do míldio e de produção dos três experimentos revelou uma interação significativa para os dois parâmetros $(\mathrm{p}<0,0001)$ em função do controle com fungicida e o início da epidemia. O desdobramento dessa interação revelou que, quando a epidemia iniciou-se aos 47 dias, o efeito do controle não interferiu na produção, enquanto que nas epidemias iniciadas aos 24 e aos 36 dias, houve um efeito altamente significativo ( $\mathrm{p}<0,0001)$ do controle. Epidemias de míldio iniciadas aos 24 e 36 dias reduziram a produção, enquanto que, quando a doença iniciou-se aos 47 dias não houve redução significativa na produção (Figura 2). Os danos à produção de frutos são inversamente proporcionais à precocidade do inicio do aparecimento dos sintomas (Figura 3) de forma que, após os 47 dias os danos se tornam insignificantes.

Maiores produções foram obtidas nas parcelas tratadas com fungicidas, sendo que, no período chuvoso, de maior vulnerabilidade das plantas, a mistura metalaxyl + mancozeb foi mais eficiente.

Este trabalho demonstrou que os aspectos relacionados com a duração, notadamente, o início da epidemia em relação ao ciclo do hospedeiro, devem ser levados em conta no
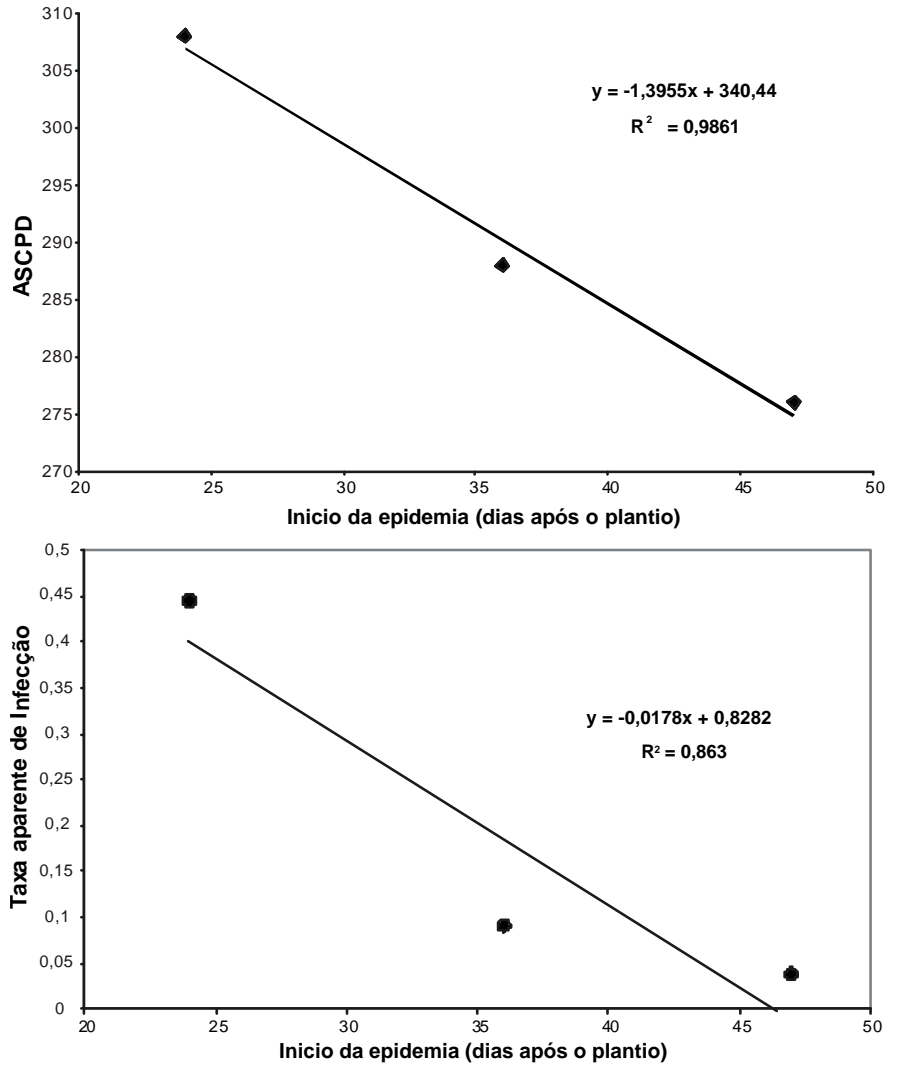

FIG. 1 - Relação entre o início da epidemia de míldio do meloeiro (Cucumis melo) e a área sobre a curva de progresso da doença (ASCPD) e a taxa aparente de infecção. Dados de três experimentos: novembro/ 2000 a janeiro/2001, fevereiro-maio/2001 (Paraipaba, CE) e fevereiro a maio/2001(Quixeré, CE). Progresso do míldio do meloeiro. Pontos representam médias de três epidemias em três experimentos distintos.

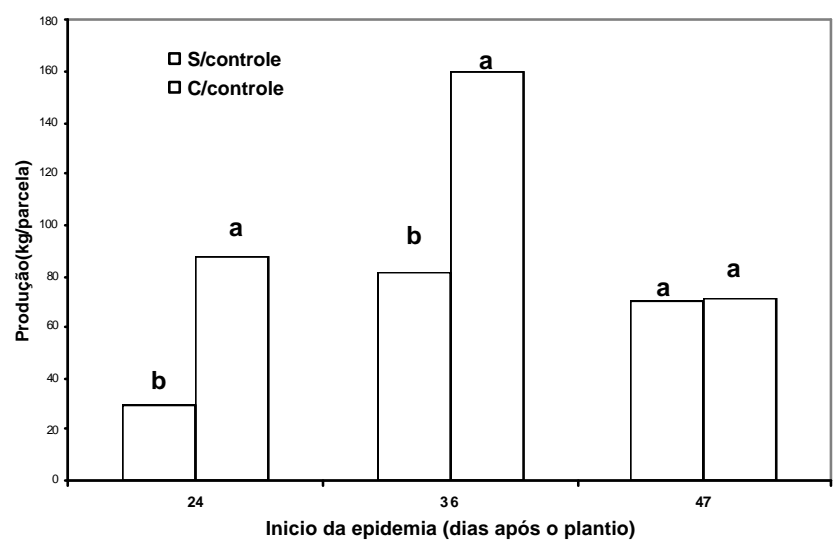

FIG. 2 - Efeito do início da epidemia do míldio na produção de frutos do meloeiro (Cucumis melo) em três experimentos sob duas pressões de doença (i. e. com e sem controle químico). Letras iguais sobre as barras agrupadas por tempo de início de epidemia não diferem estatisticamente $(\mathrm{P}=0,05)$ segundo teste de Tukey.

estabelecimento de modelos de prognósticos de danos à produção. 
Efeito do início de ocorrência do míldio na produção...

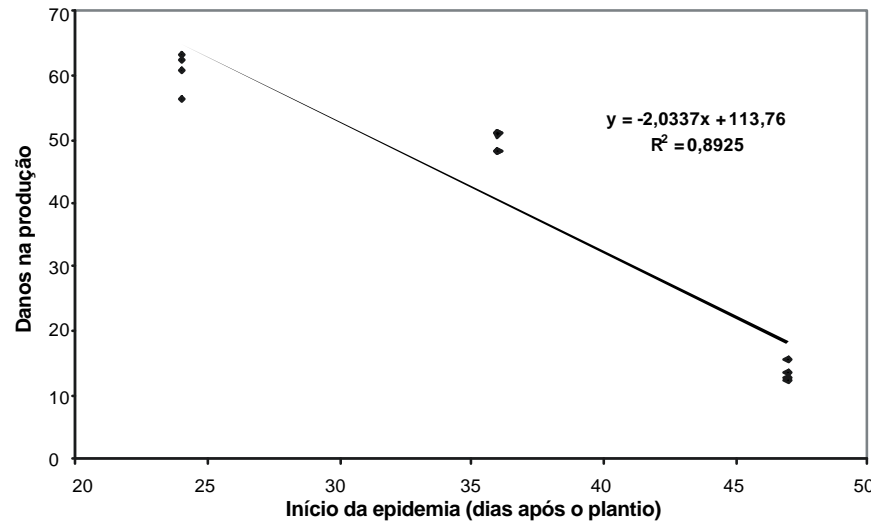

FIG. 3 - Efeito do início da epidemia de míldio causado por Pseudoperonospora cubensis nas perdas de produção de frutos do meloeiro (Cucumis melo). Pontos representam médias de 4 repetições, sendo os dados obtidos dos experimentos: A, 24 dias, B, 36 dias e C, 47 dias.

\section{REFERÊNCIAS BIBLIOGRÁFICAS}

ANUÁRIO ESTATÍSTICO DO BRASIL. Rio de Janeiro. IBGE. 1999. V. 59. 501p.

BLANCARD, D., LECOQ, H. \& PITRAT, M. Enfermedades de las cucurbitáceas: observar, identificar y luchar. Madrid: Mundi-Prensa, 1996.

CAMPBELL, C.L. \& MADDEN, L.V. Introduction to Plant Disease Epidemiology. New York John Wiley \& Sons. 1990.

CARDOSO, J.E., SANTOS, A. A. dos, VIDAL, J.C., BADU, F. O., VIANA, F. M. P. \& FREIRE, F.C.O. Eficiência de Tiofanato Metílico e Clorotalonil no controle do míldio do meloeiro. Fortaleza. Embrapa Agroindústria Tropical. Comunicado Técnico, 55. 2001.

DUSI, A.N. Melão para exportação - aspectos técnicos da produção. Brasília. DENACOOP. Publicações Técnicas, 1. 1992.
JAMES, W.C. Assessment of plant diseases and losses. Annual Review. Phytopathology 12:27-48. 1974.

KEINATH, A.P. Effect of fungicide scheduled to control gummy stem blight on yield and quality of watermelon fruit. Plant Disease 85:53-58. 2001.

KUROZAWA, C. \& PAVAN, M.A. Doenças das cucurbitáceas (abóbora, abobrinha, chuchu, melancia, melão, moranga, pepino). In: Kimati, H., Amorin, L., Bergamin Filho, A. Camargo, L.E.A. \& Rezende, J.A.M. (Eds.) Manual de Fitopatologia. São Paulo. Ceres. 1997, v.2, pp.325-337.

LATIN, R.X. Modeling the relationship between Alternaria leaf blight and yield loss in muskmelon. Plant Disease 76: 1013-1017. 1992.

LATIN, R.X., RANE, K.K. \& EVANS, J.K. Effect of Alternaria leaf blight on soluble solids content of muskmelon. Plant Disease 78:979982. 1994.

MADDEN, L.V. Measuring and modeling crop losses at the field level. Phytopatology 73: 1591-1596. 1983.

MADDEN, L.V., PENNYPACKER, S.P., ANTLE, C.E. \& KINGSOLVER, C.H.A loss model for crops. Phytopatology 71:685689. 1981.

MIDDLETON, J.T. \& WHITAKER, T.W. Some diseases of cantaloupes and honeydew melons observed in southern California. Plant Disease 30:374. 1946.

REGO, A.M. Doenças causadas por fungos em cucurbitáceas. Informe Agropecuário 17:48-54. 1995.

SANTOS, A.A., FREIRE, F.C.O., LIMA, J.A.A. \& CARDOSO, J.E. Doenças do meloeiro em áreas irrigadas no Estado do Ceará. Fortaleza. Embrapa Agroindústria Tropical. Boletim de Pesquisa, 35. 2000.

THOMAS, C.E. Downy Mildew. In: Zitter, T.A., Hopkins, D.L. \& Thomas, C.E. (Eds). Compendium of cucurbit diseases. St. Paul. The American Phytopathological Society. 1998. pp.25-27

UNIVERSIDADE FEDERAL DO CEARÁ. Recomendações de adubação e calagem para o Estado do Ceará. Fortaleza.1993. 